\title{
Polymorphie des associations au sein des forêts : Déterminants, catalogue des stations et cartographies complémentaires
}

\author{
Joseph KOULA ${ }^{* 1}$, Tié Bi TRA², Eboua N. WANDAN² \\ 'Département Mathématique-Informatique, Laboratoire de Mathématique et de Nouvelles Technologie de \\ l'Information, Institut National Polytechnique-Félix Houphouët-Boigny, BP 1093 Yamoussoukro, Côte d'Ivoire. \\ 2Département Environnement, Eaux et Forêts, Institut National Polytechnique Félix Houphouët-Boigny, BP 1093 \\ Yamoussoukro, Côte d'Ivoire. \\ ${ }^{*}$ Correspondance, courriel : koulajoseph@yahoo.fr
}

Original submitted in on 20 January 2014 . Published online at www.m.elewa.org on $31^{\text {st }}$ May 2014. http://dx.doi.org/10.4314/jab.v77i1.8

\section{RÉSUMÉ}

Objectifs : L'objectif est de proposer une démarche pragmatique pour une gestion durable d'une forêt.

Méthodologie et résultats : L'analyse descriptive multivariée des caractéristiques du sol et de la végétation de la forêt classée du Haut-Sassandra a permis de caractériser sa polymorphie et d'identifier les déterminants de celle-ci. Ungroupement des placeaux et une carte de contraintes édaphiques ont permis de dégager un catalogue des stations et une carte de recommandations de mise en valeur.

Conclusion et application: Sur sol relativement homogène, le couvert végétal de la forêt étudiée s'est révélé polymorphe essentiellement à causées activités anthropiques. Les cartes dérivées sont utiles pour une gestion rationnelle dont l'efficacité peut être accrue par couplage avec un système d'informations géographiques.

Mots-clés : Côte d'Ivoire, cartographie, Haut-Sassandra, station, Analyse statistique multivariée.

\begin{abstract}
Polymorphism of the associations in forests: Determinants, catalog of stations and additional maps Objective: The objective is to propose a pragmatic approach to sustainable forest management.

Methodology and Results: Descriptive multivariate analysis of soil and vegetation characteristics of the classified forest of Haut-Sassandra allowed characterization of its polymorphism and to identify its determinants. A grouping of plots and a map of edaphic constraints have revealed a catalog of stations and highlighted a map of recommendations.

Conclusion and application: On relatively homogeneous ground, vegetation cover of the forest studied proved polymorphic mainly due to human activities. Derived maps are useful for rational management whose effectiveness can be enhanced by coupling withal geographic information system.

Keywords: Ivory Coast, mapping, Haut-Sassandra station, Multivariate Statistical Analysis
\end{abstract}




\section{INTRODUCTION}

Les forêts jouent un rôle primordial aux plans environnemental et socio-économique. Elles interviennent de manière prépondérante dans la régulation du climat, de l'air et du cycle de l'eau. Leurs rôles dans la diminution de l'effet de serre, de la captation du carbone et du dégagement de l'oxygène est indéniable. Les forêts sont le milieu de développement idoine des cultures de rente que sont, principalement, le cacao, le café, l'hévéa et le palmier à huile. Bien qu'aujourd'hui la contribution de l'industrie du bois aux recettes d'exportation s'amenuise, elle occupait le deuxième rang après le café et avant le cacao au début des indépendances (Gibily, 1970). A travers les secteurs agricole et de l'industrie du bois, les forêts constituent une source non négligeable d'emplois et de revenus pour l'État. Les pressions anthropiques et les facteurs climatiques causent une déforestation alarmante (N'Guessan et al, 2006). De plus de 16 millions d'hectares, au début du siècle dernier, la superficie forestière de la zone

\section{MATERIEL ET METHODES}

Site de l'étude : L'étude porte sur la forêt classée du Haut-Sassandra située au nord-ouest du département de Daloa, dans le centre-ouest de la Côte d'Ivoire. Elle est située entre $6^{\circ} 51^{\prime}$ et $7^{\circ} 24^{\prime}$ de latitude Nord, $6^{\circ} 59^{\prime}$ et $7^{\circ} 10^{\prime}$ de longitude ouest (Figure 1).D'une superficie de 102400 ha et limitée à l'ouest par le fleuve Sassandra, c'est une forêt semi-décidue à Triplochyton, Monsonia altissima, Celtis sp. avec dans son extrême nord-ouest, une savane incluse à Loudetia simplex, Andropogon macrophyllus, dont la strate arborée est dominée par Lophira lanceolataet Terminalia glaucescens (Kouamé, 1998). Les sols le long du fleuve sont essentiellement hydromorphes à gley et pseudogley et peu évolués. Le reste de la forêt est sur sols ferralitiques remaniés indurés sur granites dans la partie nord du massif; rajeunis avec inselberg au centre; et à recouvrement dans sa partie sud-est (Oswald, 2005) dense humide a été estimée à la fin du même siècle à moins de 4 millions d'hectares (Yao, 1999).En moins d'un demi-siècle, le couvert forestier ivoirien s'est dégradé à plus de $75 \%(\mathrm{~N}$ 'Da et al, 2008) et est estimé de nos jours à moins de 2,50 ha. Malgré les dispositions législatives et administratives pour le renforcement de la conservation des aires protégées, le domaine forestier de l'État reste très menacé. II se présente aujourd'hui sous des formes variées allant de la forêt riche en équilibre à la forêt ruinée en culture ou en jachère. L'objectif principal est de proposer aux gestionnaires de forêts en général et des forêts classées en particulier, une démarche pragmatique débouchant sur des outils de décisions pour une gestion rationnelle aux fins de la pérennisation des forêts classées. II s'agit spécifiquement d'identifier les déterminants de la polymorphie des associations; de dégager une carte de mise en valeur à partir d'une carte de contraintes et d'un catalogue de stations.

\section{Données de l'étude}

Collecte des données : Dans le cadre d'un inventaire général à $1,25 \%$, la forêt a été parcourue du Sud au Nord par 29 layons, numérotés d'Est en Ouest, de 25 $\mathrm{m}$ de large et distants de $2 \mathrm{~km}$ à l'exception des 2 derniers séparés de $1,50 \mathrm{~km}$. Des placeaux de 0,50 ha (200 m x 25m) ont été disposés le long des layons ; leur nombre varie entre 75 et 102 selon les layons. La forêt a donc été subdivisée en 2533 placeaux $P_{i j}$ non géoréférencés avec $j$ le numéro d'ordre au sein du layoni. Sur chaque placeau, des échantillons de sol ont été prélevés et analysés; des observations et des mesures ont été faites sur le couvert végétal; les essences forestières de même que leurs diamètres ont été également répertoriés. 


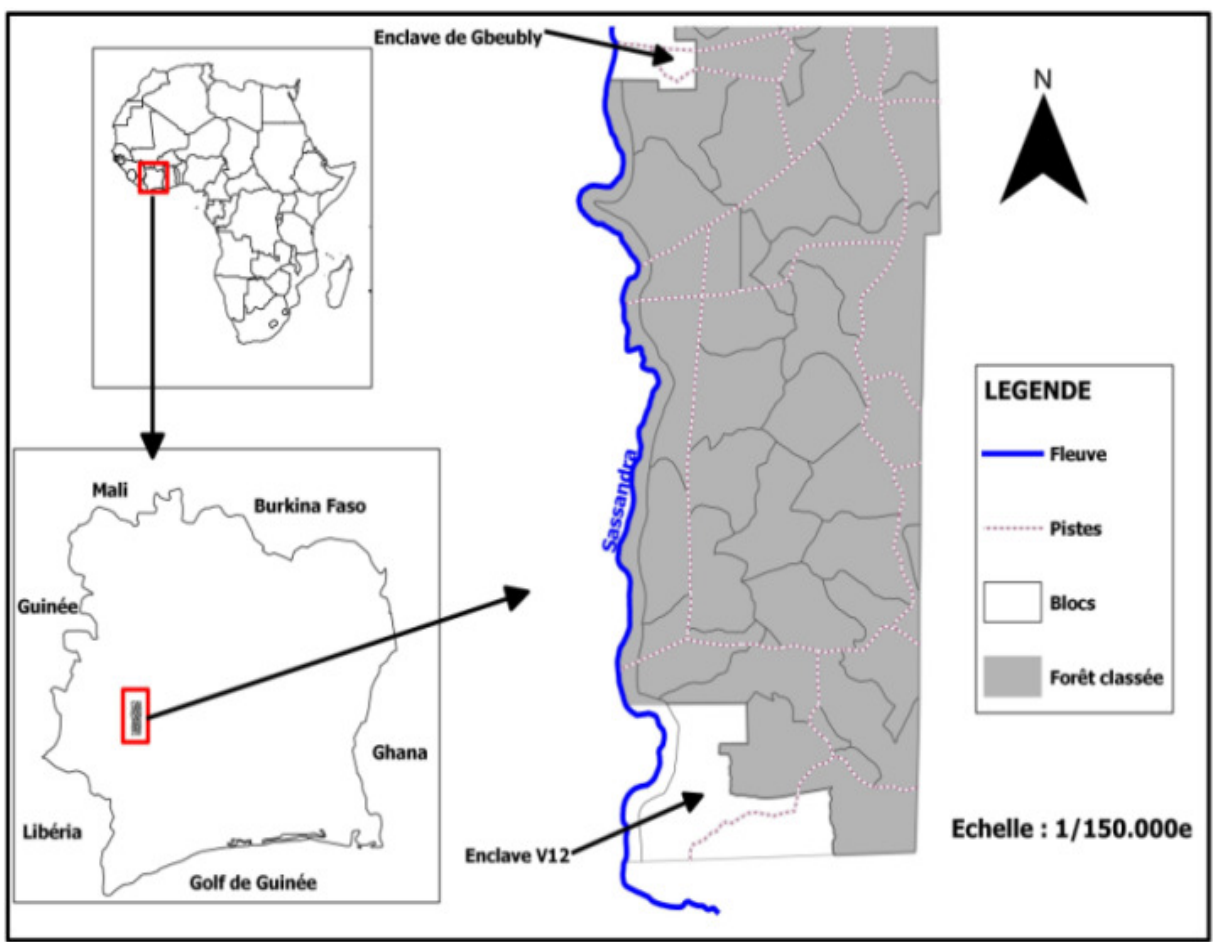

Figure 1 : Carte de situation de la forêt classée du Haut Sassandra

Classes de diamètres et typologie des peuplements: Trois classes des diamètres des essences primaires $(P)$ ont été définies: les peuplements en régénération, d'avenir et exploitables avec pour seuils en tige de $\mathrm{P}$ par hectare de 125,30 et 8 , respectivement. Une variable typologie des peuplements (TY), a été synthétisée et codifiée de 1 à 8 (Tableau 1) sur la base dela richesse des placeaux en $\mathrm{P}$ de différentes classes de diamètre.
Caractérisation des sols et de la végétation: $A u$ niveau du sol, l'hydromorphie $(\mathrm{H})$, la texture $(\mathrm{T})$, la présence de gravillons dans les dix premiers centimètres $(G)$ et la présence d'affleurements rocheux et de croûte latéritique $(C)$ ont été déterminées. Pour les formations végétales, les taux de recouvrement des strates supérieures (SS) et moyenne (SM), la formation végétale au sol (S), et le type de cultures portées $(C P)$ ont été recueillies.

Tableau 1 : Typologie des peuplements

\begin{tabular}{|c|c|c|c|l|}
\hline Régénération & Peuplement & Peuplement & \multicolumn{2}{|c|}{ Typologie des Peuplements } \\
\cline { 4 - 5 } $5 \leq \Phi<20$ & $20 \leq \Phi<50$ & $50 \leq \Phi$ & Codes & Description \\
\hline+ & + & + & $\mathrm{A}[1]$ & Riche en équilibre \\
\hline+ & - & + & $\mathrm{B}[2]$ & Riche avec déficit en bois moyens \\
\hline- & + & + & $\mathrm{C}[3]$ & Riche avec déficit en régénération \\
\hline- & - & + & $\mathrm{D}[4]$ & Dégradé avec déficit en bois moyens et régénération \\
\hline+ & + & - & $\mathrm{E}[5]$ & Riche avec déficit en gros bois \\
\hline- & + & - & $\mathrm{F}[6]$ & Dégradé avec déficit en gros bois et régénération \\
\hline+ & - & - & $\mathrm{G}[7]$ & Dégradé avec déficit en gros bois et bois moyen \\
\hline- & - & - & $\mathrm{H}[8]$ & Ruiné, friche ou culture \\
\hline
\end{tabular}

${ }^{\dagger}:+$ : Seuil atteint ; - Seuil non atteint ; $\Phi:$ Diamètre des essences primaires $P 1$ en centimètres 
Leur codage est consigné dans le tableau 2. Les effectifs des essences primaires, secondaires et tertiaires ont été également recueillis. Sur les 2533 placeaux inventoriés, 2348 placeaux sans données manquantes ont été effectivement utilisés.

\section{Méthodes}

Le modèle Statistique: La distribution spatiale des données implique le modèle de base suivant: Soit $s \in \mathfrak{R}^{2}$ les coordonnées d'un placeau ou point dans un espace euclidien de dimension 2 qu'est la forêt étudiée. Les coordonnées sont le numéro du layon $i(1 \leq i \leq l)$ le numéro d'ordre $j\left(1 \leq j \leq n_{i}\right)$ où lreprésentent le nombre total de layon, $n_{i}$ le nombre de placeaux dans le layon $t$. .
La variable aléatoire $Z(s)$ représente un vecteur des observations possibles sur les différents descripteurs au niveau du placeau $p_{s} \equiv p_{i j}$. $s$ variant dans l'ensemble des indices $D \subset \Re^{2}, Z(s)$ est assimilable à un processus stochastique: $\{Z(s): s \in D\}$. $D$ étant un sous-ensemble non-aléatoire de $\Re^{2}$, le modèle est géostatistique avec $Z(s)$ échantillonné en des points ou placeaux fixes. Dans ce type de modèle, $Z\left(s_{k}\right)$ et $Z\left(s_{r}\right)$ avec $k \neq r$ sont souvent corrélés lorsqu'ils sont dans le voisinage l'un de l'autre. II n'est aussi pas judicieux d'affirmer, sans justification, l'indépendance des erreurs. Les inférences sur $Z(s)$ requièrent des conditions de stationnarité comme dans les séries chronologiques.

Tableau 2 : Codification des caractéristiques des sols et de la végétation

\begin{tabular}{|c|c|c|c|}
\hline Milieu & Paramètres & Description & Code \\
\hline \multirow{10}{*}{ Sol } & \multirow{2}{*}{ Hydromorphie(H) } & Sol ferme & 0 \\
\hline & & $\begin{array}{l}\text { Sol temporairement ou permanence } \\
\text { marécageux }\end{array}$ & 1 \\
\hline & \multirow[t]{4}{*}{ Texture $(T)$} & Sol peu profond & 0 \\
\hline & & Sol sableux à argileux-sableux & 1 \\
\hline & & Sol argileux-sableux & 2 \\
\hline & & Sol argileux & 3 \\
\hline & \multirow{2}{*}{$\begin{array}{l}\text { Gravillons dans les } 10 \text { premiers } \\
\text { centimètres }(G)\end{array}$} & Peu ou pas présents & 0 \\
\hline & & Présents & 1 \\
\hline & $\begin{array}{l}\text { Affleurements rocheux et de croûte } \\
\text { latéritique }(\boldsymbol{C})\end{array}$ & Absence & 0 \\
\hline & & Présence & 1 \\
\hline \multirow{12}{*}{$\begin{array}{l}\text { Formations } \\
\text { végétales }\end{array}$} & \multirow{3}{*}{$\begin{array}{l}\text { Taux de recouvrement des strates } \\
\text { supérieures(SS) }\end{array}$} & $10 \%<S S<30 \%$ & 1 \\
\hline & & $30 \%<S S<70 \%$ & 2 \\
\hline & & $70 \%<$ SS & 3 \\
\hline & \multirow{3}{*}{$\begin{array}{l}\text { Taux de recouvrement des strates } \\
\text { moyennes(SM) }\end{array}$} & $10 \%<\mathrm{SM}<30 \%$ & 1 \\
\hline & & $30 \%<\mathrm{SM}<70 \%$ & 2 \\
\hline & & $70 \%<$ SM & 3 \\
\hline & \multirow[t]{6}{*}{ Cultures portées(CP) } & Pas de culture & 0 \\
\hline & & Culture vivrière pure & 1 \\
\hline & & Culture pérenne lâche mal entretenue (jeune) & 2 \\
\hline & & $\begin{array}{l}\text { Culture pérenne lâche bien entretenue } \\
\text { (jeune) }\end{array}$ & 3 \\
\hline & & $\begin{array}{l}\text { Culture pérenne lâche mal entretenue (en } \\
\text { production) }\end{array}$ & 4 \\
\hline & & $\begin{array}{l}\text { Culture pérenne lâche bien entretenue (en } \\
\text { production) }\end{array}$ & 5 \\
\hline
\end{tabular}

Caractérisation de la Forêt: La caractérisation générale de la forêt repose sur la dérivation d'un placeau-type qui correspond au « placeau modal », pas nécessairement observable et parfaitement décrit par 
les valeurs les plus fréquentes des descripteurs. II est mieux indiqué que celui basé sur les valeurs moyennes à cause de la non-normalité et la nature discrète des descripteurs. Le sens et le degré d'association des valeurs des descripteurs nécessaires seront appréciés grâce au coefficient de corrélation de Spearman ici préféré au coefficient de corrélation linéaire de Pearson.

Principaux déterminants de la polymorphie de la forêt: L'identification des déterminants les plus pertinents de la polymorphie de la forêt a été faite par l'analyse en composantes principales (ACP) utilisant la matrice de variance-covariance à cause de l'absence d'unités pour les descripteurs (Morisson, 1990; Legendre et Legendre, 1979). La qualité des données conforte l'utilisation de l'ACP même si les données n'ont pas une distribution multi normale (Ibanez, 1971). La procédure PRINCOMP du logiciel Statistical Analysis System (SAS) a été ici utilisée.

Groupements statistiques des placeaux: Plusieurs méthodes et algorithmes de groupements existent (Hartigan, 1975 ; Gower, 1967).Elles opèrent soit sur la base de règles dépendant de critères liés à la spécification a priori ou non des groupes soit selon le but poursuivi. Les méthodes de groupement non hiérarchique ont été ici préférées aux méthodes discriminantes car elles produisent une seule partition et optimisent l'homogénéité intragroupe contrairement

\section{RÉSULTATS ET INTERPRÉTATION}

Caractérisation générale de la Forêt Classée du Haut-Sassandra: Les valeurs modales des descripteurs évaluées sur la base des 2348 placeaux (Tableau 3) révèlent que la forêt classée du HautSassandra est à majorité une forêt :

ruinée, en friche ou en culture (mode de TY = 8) : avec un déficit en régénération et en peuplements d'avenir et exploitables; d'où sa faible valeur économique dont l'accroissement nécessite un reboisement et une gestion rationnelle des peuplements d'avenir et exploitables ;

- $\quad$ sursols fermes argilo-sableux (modes: $\mathrm{H}=0$, $\mathrm{T}=2$ ); avec peu ou pas de gravillons (mode $\mathrm{G}=0$ ); avec absence d'affleurements rocheux ou de croûte latéritique (Mode $\mathrm{C}=0$ ). Ceci suggère un potentiel certain pour un reboisement avec un large éventail d'essences: aux méthodes hiérarchiques qui produisent des groupes non nécessairement disjoints et requiert la présence d'une variable de classification, la normalité et la continuité des descripteurs, conditions trop contraignantes pour les données disponibles. La procédure FASCLUS de SAS basée sur la méthode des $k$ moyennes a été ici utilisée avec pour option le nombre de groupe issu de l'ACP. Bien que ne comparant pas toutes les partitions possibles, elle produit un groupement acceptable dans la majorité des cas (Wilkinson et al. 2004). Pour une analyse complète trois (3) scénarii ont été spécifiés en combinant les groupes de descripteurs plutôt que les descripteurs pris individuellement. Le Scénario I est basé sur les données pédologiques $(H, T, G, C)$. Le Scénario II est constitué des descripteurs du scénario I auxquels est adjointe la typologie(TY) des peuplements alors que le Scénario III est identique au scénario I auquel ont été intégrés les autres caractères de la formation végétale que sont S, SS, SM et CU.

Cartographies complémentaires: Les données utilisées sont rattachées aux placeaux d'inventaire. Des cartes par point seront générées à l'aide du logiciel Mapinfo. Chaque placeau représente une surface élémentaire de la forêt classée, un rectangle de largeur 200 mètres et de longueur la distance entre deux layons, soit 40 hectares. Les données manquantes ont été extrapolées depuis le voisinage.

- une forêt avec SS et SM comprises respectivement entre $10 \%$ et $30 \%, 30 \%$ et $70 \%$; sans culture (Mode de $\mathrm{CU}=0$ ) : la forêt étudiée a pu être convenablement préservée des infiltrations.

La forêt étudiée se révèle donc être une forêt polymorphe : riche, dégradée et ruinée respectivement sur $34 \%, 34 \%$ et $32 \%$ de son étendue sur sol essentiellement argilo-sableux sans éléments grossiers.

Liens entre les caractéristiques de la Forêt Classée du Haut-Sassandra: Les résultats de l'analyse de corrélation (Tableau 3) sont conformes, pour la plupart, aux relations attendues: les descripteurs du sol sont significativement ( $T$ et $H$ ) ou très hautement significativement (les autres paires) corrélés. Les descripteurs $\mathrm{G}$ et $\mathrm{C}$ qui prennent en compte les croûtes de cuirasse et les éléments grossiers sont très fortement et positivement corrélés. 
Koula et al. J. Appl. Biosci. 2014. Polymorphie des associations au sein des forets : Déterminants, Catalogue des stations et cartographies complémentaires.

Tableau 3: Corrélations entre les descripteurs du sol et de la formation végétale

\begin{tabular}{|c|c|c|c|c|c|c|c|c|c|c|}
\hline \multirow{2}{*}{\multicolumn{2}{|c|}{$\begin{array}{c}\text { Descripteur } \\
\text { [Mode] }\end{array}$}} & \multicolumn{4}{|c|}{ Descripteurs du sol } & \multicolumn{5}{|c|}{ Descripteurs de la formation végétale } \\
\hline & & \multirow{2}{*}{$\begin{array}{c}\mathbf{H} \\
1,000 \\
(0)^{\star *}\end{array}$} & \multirow[t]{2}{*}{$\mathrm{T}$} & \multirow[t]{2}{*}{ G } & \multirow[t]{2}{*}{ C } & \multirow[t]{2}{*}{ TY } & \multirow[t]{2}{*}{$\mathbf{S}$} & \multirow[t]{2}{*}{ SS } & \multirow[t]{2}{*}{ SM } & \multirow[t]{2}{*}{ CU } \\
\hline ర్ల్ర & $\begin{array}{l}H \\
{[0]^{*}}\end{array}$ & & & & & & & & & \\
\hline$\stackrel{\bar{\Phi}}{5}$ & $\begin{array}{l}T \\
{[2]}\end{array}$ & $\begin{array}{l}-0,046 \\
(0,026)\end{array}$ & $\begin{array}{c}1,000 \\
(0)\end{array}$ & & & & & & & \\
\hline 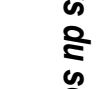 & $\begin{array}{l}G \\
{[0]}\end{array}$ & $\begin{array}{c}-0,442 \\
(0)\end{array}$ & $\begin{array}{c}-0,104 \\
(0)\end{array}$ & $\begin{array}{c}1,000 \\
(0)\end{array}$ & & & & & & \\
\hline 은 & $\begin{array}{l}C \\
{[0]}\end{array}$ & $\begin{array}{c}-0,265 \\
(0)\end{array}$ & $\begin{array}{c}-0,228 \\
(0)\end{array}$ & $\begin{array}{c}0,597 \\
(0)\end{array}$ & $\begin{array}{c}1,000 \\
(0)\end{array}$ & & & & & \\
\hline 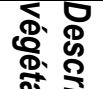 & $\begin{array}{l}T Y \\
{[8]}\end{array}$ & $\begin{array}{l}-0,004 \\
(0,851)\end{array}$ & $\begin{array}{l}-0,071 \\
(0,001)\end{array}$ & $\begin{array}{c}0,004 \\
(0,8863)\end{array}$ & $\begin{array}{c}0,004 \\
(0,843)\end{array}$ & $\begin{array}{c}1,000 \\
(0)\end{array}$ & & & & \\
\hline$\frac{0}{\frac{D}{8}}$ & $\begin{array}{l}S \\
{[2]}\end{array}$ & $\begin{array}{c}0,024 \\
(0,254)\end{array}$ & $\begin{array}{l}-0,012 \\
(0,544)\end{array}$ & $\begin{array}{l}-0,020 \\
(0,328)\end{array}$ & $\begin{array}{l}-0,015 \\
(0,479)\end{array}$ & $\begin{array}{c}0,249 \\
(0)\end{array}$ & $\begin{array}{c}1,000 \\
(0)\end{array}$ & & & \\
\hline$\frac{2}{8}$ & $\begin{array}{l}\text { SS } \\
{[1]}\end{array}$ & $\begin{array}{l}-0,005 \\
(0,823) \\
\end{array}$ & $\begin{array}{c}0,120 \\
(0)\end{array}$ & $\begin{array}{l}-0,040 \\
(0,052)\end{array}$ & $\begin{array}{l}-0,032 \\
(0,107)\end{array}$ & $\begin{array}{l}-0,047 \\
(0,023) \\
\end{array}$ & $\begin{array}{c}-0,241 \\
(0)\end{array}$ & $\begin{array}{c}1,000 \\
(0)\end{array}$ & & \\
\hline$\stackrel{\infty}{s}$ & $\begin{array}{l}S M \\
{[2]}\end{array}$ & $\begin{array}{l}-0,026 \\
(0,203)\end{array}$ & $\begin{array}{c}0,079 \\
(0)\end{array}$ & $\begin{array}{c}-0,007 \\
(0,734)\end{array}$ & $\begin{array}{c}-0,034 \\
(0\end{array}$ & $\begin{array}{c}-0,073 \\
(0)\end{array}$ & $\begin{array}{c}-0,263 \\
(0)\end{array}$ & $\begin{array}{c}0,393 \\
(0)\end{array}$ & $\begin{array}{c}1,000 \\
(0)\end{array}$ & \\
\hline$\stackrel{\#}{\tilde{5}}$ & $\begin{array}{l}C U \\
{[0]}\end{array}$ & $\begin{array}{l}-0,001 \\
(0,613)\end{array}$ & $\begin{array}{c}0,009 \\
(0,619)\end{array}$ & $\begin{array}{c}-0,007 \\
(0,730)\end{array}$ & $\begin{array}{l}-0,009 \\
(0,669)\end{array}$ & $\begin{array}{c}0,032 \\
(0,120)\end{array}$ & $\begin{array}{c}0,126 \\
(0)\end{array}$ & $\begin{array}{l}-0,069 \\
(0,001)\end{array}$ & $\begin{array}{c}-0,074 \\
(0)\end{array}$ & $\begin{array}{c}1,000 \\
(0)\end{array}$ \\
\hline
\end{tabular}

${ }^{*}:$ Mode des descripteurs ; ** : P-value du test bilatéral de nullité du coefficient de corrélation

Parmi les descripteurs de la formation végétale seule la culture portée et la typologie ne sont évidemment pas linéairement corrélés: la typologie est construite en utilisant uniquement les essences primaires. Tous les autres descripteurs de la formation végétale sont corrélés (TY et SS) ou très fortement corrélés (cas des autres paires) lorsque pris deux à deux. Seule T, parmi les descripteurs du sol, est liée aux descripteurs de la formation végétale que sont TY et SS et SM. L'absence de relation entrela «fertilité intrinsèque » (caractéristiques du sol) et la fertilité exprimée (caractéristiques de la formation végétale) pourrait être due à l'effet des actions humaines sur la flore. En effet, les actions anthropiques telles que la coupe d'essences commercialisables et la création de plantations semblent plus prépondérantes dans la détermination de l'état actuelle de la forêt que la nature du sol.

Variabilité au sein de la forêt classée du HautSassandra : Les sols rencontrés dans la forêt classée du Haut-Sassandra sont assez homogènes (variance de 0,65). Sur la base des descripteurs du sol et de la formation végétale autre que TY (Scénario III) la forêt est relativement homogène (variance de 2,33.) (Tableau IV). Ensuite, l'analyse révèle une grande variabilité (variance multivariée de 6,42) lorsque l'on tient compte de leur potentialités intrinsèque (descripteurs du sol) et exprimées nettes des effets climatiques et humains (Typologie); comme dans le scénario II. Cette variabilité est essentiellement due à TY, principal déterminant de l'axe principal 1, qui explique environ $90 \%$ de la variance totale.

Groupement des placeaux : Sur la base du Scénario I, 8 types de sol ont été dégagés: types 1300 (7 placeaux) ; 1200 (574 placeaux) ; 1100 (57 placeaux) ; 0200 (900 placeaux); 1210 (1 placeau); 0210 (438 placeaux) ; 0211 (331 placeaux) ; 0011 (40 placeaux). Les placeaux à sols argilo sableux (types $0200 ; 0210$; 0211 ; 1200 ; 1210) représentent plus de 95\% des placeaux analysés confirmant ainsi l'homogénéité des sols 
Koula et al. J. Appl. Biosci. 2014. Polymorphie des associations au sein des forets : Déterminants, Catalogue des stations et cartographies complémentaires.

Tableau 4 : Contributions des descripteurs à la formation des axes principaux

\begin{tabular}{|c|c|c|c|c|c|c|c|c|c|c|c|c|c|}
\hline \multirow{2}{*}{$\begin{array}{l}\text { Scenario } \\
\text { [variance] }\end{array}$} & \multirow[b]{2}{*}{ Axes } & \multicolumn{3}{|c|}{ Variance expliquée } & \multicolumn{4}{|c|}{ Descripteurs du Sol } & \multicolumn{5}{|c|}{ Descripteurs de la Forrnation Végétale } \\
\hline & & Valeur & $\%$ & $\begin{array}{c}\text { Cumu } \\
1\end{array}$ & $\mathrm{H}$ & $\mathrm{T}$ & G & $\mathrm{C}$ & TY & $\mathrm{s}$ & ss & SM & $\mathrm{CU}$ \\
\hline \multirow{3}{*}{$\begin{array}{c}\text { I } \\
{[0,6497]}\end{array}$} & Axe1 & 0,3595 & 0,55 & 0,55 & $-0,53$ & $-0,07$ & 0,07 & 0,44 & & & & & \\
\hline & Axe2 & 0,1443 & 0,22 & 0,77 & 0,77 & $-0,38$ & 0,27 & 0,43 & & & & & \\
\hline & Axe3 & 0,0862 & 0,13 & 0,91 & 0,34 & 0,85 & 0,40 & $-0,11$ & & & & & \\
\hline \multirow{5}{*}{$\begin{array}{c}\text { II } \\
{[6,4252]}\end{array}$} & Axel & 57773 & 0,05 & 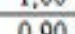 & 0,01 & 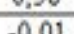 & 0,01 & 0 & 000 & & & & \\
\hline & Axe2 & 0,3585 & 0,05 & 0,95 & $-0,53$ & $-0,07$ & 0,72 & 0,44 & $-0,01$ & & & & \\
\hline & Axe3 & 0,1442 & 0,02 & 0,98 & 0.77 & $-0,38$ & 0,28 & 0.43 & 0 & & & & \\
\hline & Axe4 & 0,0856 & 0,01 & 0,99 & 0,33 & 0,84 & 0,40 & $-0,12$ & 0,01 & & & & \\
\hline & Axes & 0,9985 & 0,01 & 1,00 & $-0,07$ & 0,37 & $-0,49$ & 0,78 & 0 & & & & \\
\hline \multirow{4}{*}{ III } & Axe1 & 0,9138 & 0,39 & 0,39 & 0,01 & $-0,02$ & $-0,01$ & 0 & & 0,90 & $-0,31$ & $-0,29$ & 0,06 \\
\hline & Axe2 & 0,4231 & 0,18 & 0,57 & 0,11 & 0,08 & 0,08 & $-0,13$ & & 0,41 & 0,67 & 0,56 & $-0,01$ \\
\hline & Axe3 & 0,3550 & 0,15 & 0,72 & 0,52 & $-0,05$ & $-0,05$ & 0,42 & & 0,12 & 0,15 & 0,17 & 0 \\
\hline & Axe4 & 0,2197 & 0,09 & 0,82 & 0,03 & $-0,03$ & $-0,03$ & $-0,03$ & & 0,02 & $-0,65$ & 0,75 & $-0,01$ \\
\hline \multirow{4}{*}[2,3333]{} & Axe5 & 0.1443 & 0.06 & 0.88 & 0.73 & $-0,34$ & 0.26 & 0,40 & & 0.03 & 0.05 & 0.02 & $-0,32$ \\
\hline & Axe6 & 0,1331 & 0,06 & 0,94 & 0,25 & $-0,10$ & 0,09 & 0,14 & & $-0,05$ & 0,03 & 0,03 & 0,94 \\
\hline & Axe7 & 0,0853 & 0,04 & 0,97 & 0,32 & 0,85 & 0,40 & $-0,13$ & & $-0,01$ & $-0,05$ & $-0,02$ & $-0,01$ \\
\hline & Axe8 & 0,0594 & 0,02 & 1,00 & $-0,07$ & 0,38 & $-0,49$ & 0,78 & & 0 & $-0,03$ & 0,01 & $-0,01$ \\
\hline
\end{tabular}

Le scénario II produit 49 groupes (entre 1 et 280 placeaux chacun) comparativement au scénario III qui en produit 70. Dans la perspective d'établissement de catalogue de stations, de recommandations et de carte de mise en valeur le groupement issu du scénario II semble le plus prometteur de par ses descripteurs et le nombre relativement petit des groupes qui en sont issus.

Établissement de la Carte de Contraintes au Reboisement : La finalité d'un reboisement se résume essentiellement en une optimisation tant au niveau financier qu'écologique de la forêt. L'on suppose ici que seuls le peuplement existant et le type de sol constituent les éléments majeurs de la stratégie de reboisement

Hiérarchisation des contraintes édaphiques :Les 49 groupes statistiques se répartissent en huit (8) catégories de sol définies par les critères $\mathrm{H}, \mathrm{T}, \mathrm{G}$ et $\mathrm{C}$. La profondeur du sol n'est limitant qu'en sommet d'interfluve où les affleurements de roches ou de cuirasse sont fréquents. Le cas échéant, la matrice terreuse est peu épaisse et souvent gravillonnaire (sol de type 0011). La sélection naturelle des essences est alors stricte: seules des espèces à enracinement superficiel peuvent s'y développer en atteignant rarement le dernier stade. Les sols avec des croûtes de cuirasse et des éléments grossiers (0211) font suite sur le versant, vers l'aval, aux sols peu profonds. La profondeur n'est pas ici limitant contrairement à la présence d'éléments grossiers qui induisent des déformations aux racines (coudes, balais de sorcières,...) faisant ainsi obstacle à l'élongation des racines, notamment le pivot, organe essentiel dans l'encrage du végétal. L'influence de l'hydromorphie est tant d'ordre physique que physico-chimique et physiologique. L'hydromorphie limite l'aération, l'activité biologique d'humification et de minéralisation. Lorsqu'en certaines situations (partie amont des basversants), elle est modérée, l'aération et l'humidité sont assurées presque en toute saison; ce qui favorise le développement de la végétation. C'est le cas pour la majorité des sols du type 1200 . Toute fois la permanence de l'hydromorphie qui dépend également de la texture du sol est d'autant plus marquée et persistante que le sol est argileux; ce qui restreint considérablement les possibilités de mise en valeur.

La particularité des sols de type 1210 réside dans la présence d'éléments grossiers. Si ceux-ci sont en proportion élevés, cas de figure plutôt rare, le drainage devient encore plus déficient. Ceci rapprocherait leur comportement vis-à-vis de l'eau de celui des sols hydromorphes argileux (1300).La nature du système racinaire joue un rôle prépondérant dans le reboisement. Les lignes dela Figure 3 synthétisent la hiérarchisation des contraintes en ce sens. Les recommandations se fondent sur le fait que les essences à pivot pérenne sont à la fois sensibles au gravillonnement et à l'hydromorphie. Par contre la disponibilité en eau est la contrainte essentielle pour les espèces sans pivot. Par exemple, le Bossé, Guarea Cedrata (Meliaceae);le Lingué, Afzélia africana (Ceasalpiniaceae) ;'Oba, Bombas buonopozense 
Koula et al. J. Appl. Biosci. 2014. Polymorphie des associations au sein des forets : Déterminants, Catalogue des stations et cartographies complémentaires.

(Bombacaceae) ; le Samba, Triplochiton scleroxylon (Sterculiaceae) qui sont des espèces à systèmes racinaires traçant sont à conseiller pour des sols de type 0211 à contraintes sévères alors que les espèces à système racinaires mixtes tels que le $\mathrm{Bi}$, Eribroma oblongum (Sterculiacea); le Koto,Pterygota macrocarpa (Sterculiaceae) etle Tiama, Entandrophragma angolense (Meliaceae) conviennent aux sols de type 0210 à contraintes modérées. Pour les sols à contraintes éliminatoires (type 1210), en tenant compte de la sévérité de l'hydromorphie, les espèces locales ou exotiques adaptées comme le Gmelinaarborea (Lamiacea) doivent être envisagées.

La carte des contraintes :Les cinq niveaux de contraintes ci-dessus définissent 5 strates cartographiques: la strate à contraintes au reboisement nulles sur $38,30 \%$ de la superficie; la strate à contraintes modérées $(18,70 \%)$; la strate à contraintes sévères $(14,10 \%)$; la strate à contraintes très sévères $(24,50 \%)$ et la strate à contraintes éliminatoires $(4,40 \%)$. Les proportions ont été calculées sur la base de la zone réellement inventoriée, soit environ 94000 hectares (hormis les 2 enclaves). Les sols à contraintes éliminatoires sont essentiellement localisés dans la moitié nord du massif, dans deux courbures du fleuve Sassandra. II n'apparaît aucun gradient de séverité de contraintes au sein de la forêt (Figure 2).

\section{Recommandations et Carte de Mise en Valeur}

État Actuel de la Forêt : La valeur forestière rendue par TY a été hiérarchisée en 4 groupes, rendant compte des principales orientations sylvicoles à retenir : forêt naturelle en équilibre (type $A$ ); retour à l'équilibre pour la forêt naturelle grâce à une sylviculture adaptée (types B, C, E) ; enrichissement de la forêt naturelle (types $F$ et $G$ ) ; recours à la plantation en plein (types $D$ et $H$ ) précédée d'une exploitation (type D).

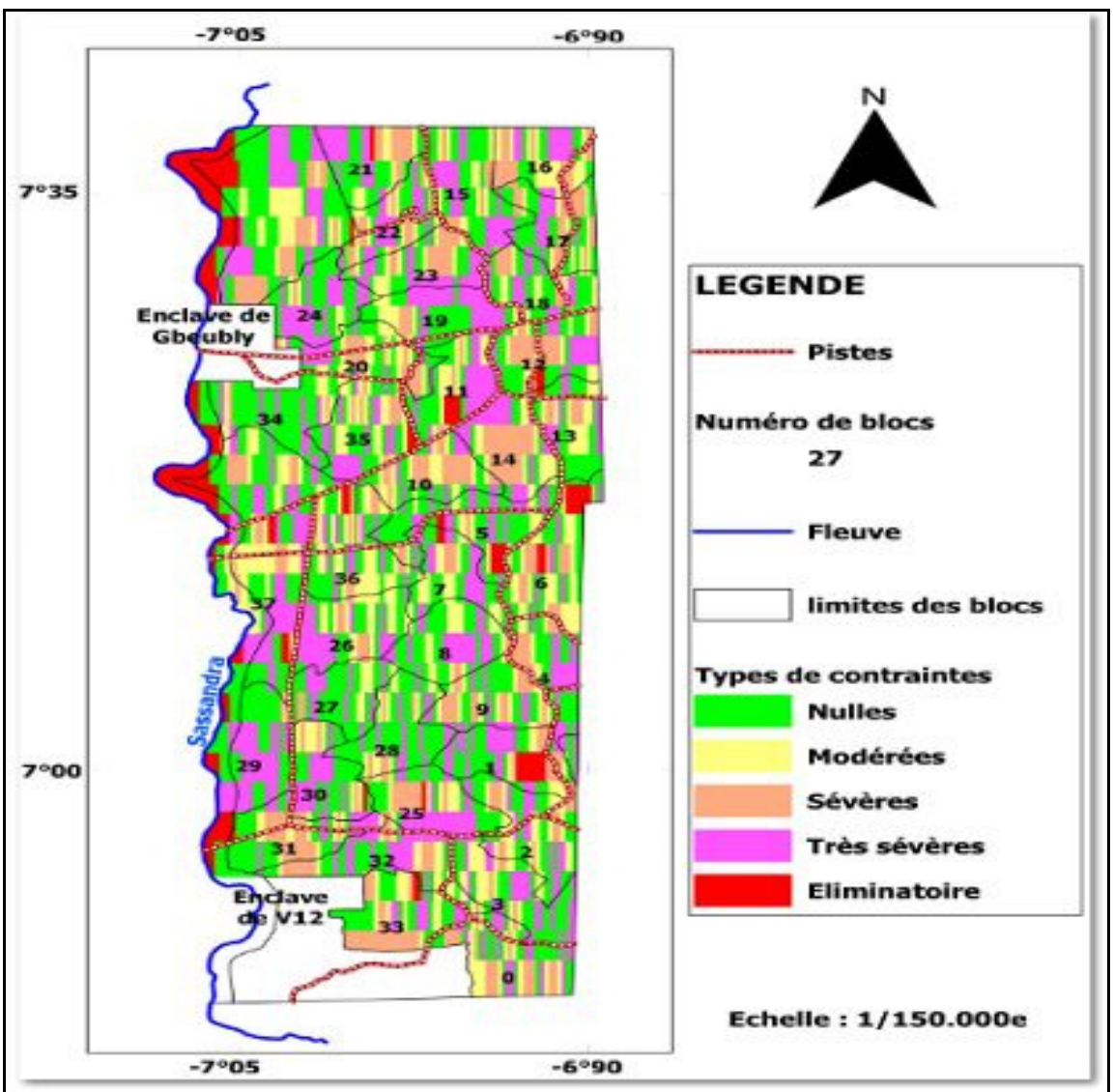

Figure 2. Carte de contraintes au reboisement dans la forêt classée du Haut-Sassandra

Une grille d'analyse générée par le croisement des orientations sylvicoles et des contraintes édaphiques au reboisement (Figure 3) permet d'identifier six (6) types de recommandations : 
- La recommandation de type 1 (34,40\% de la forêt) concerne toutes les zones de forêt naturelle riche, où l'on recherchera simplement un retour à l'équilibre du peuplement.

- Les recommandations de type 2, 3 et 4 concernent des zones nécessitant des travaux de reboisement, par enrichissement ou en plein selon l'état de dégradation, et avec un cortège d'essences différent selon les contraintes édaphiques. Ainsi, les recommandations de type 2 concernent la zone à toute sorte d'espèces $(24,96 \%)$; les espèces à système racinaire mixte sont conseillées pour $12,40 \%$ de la forêt (recommandation de type 3) ; ceux de type 4 reposent essentiellement sur les espèces à système racinaire traçant $(9,40 \%)$;

Le type $5(13,8 \%$ du massif) concerne des travaux de reboisement en plein avec des essences supportant une certaine hydromorphie. En ce sens Gmelina arborea a été proposé. et ce type n'a donc pas été étendu aux zones simplement à enrichir.

- Le type 6 qui concerne $5 \%$ de la forêt représente des faciès dégradés même ruinés, mais dont les contraintes interdisent toute action de reboisement.

Les essences peuvent être réparties en espèces principales et en espèces diverses. Les essences principales se subdivisent en trois catégories en fonction de leur fréquence de commercialisation. Ainsi, la catégorie $1 \quad\left(P_{1}\right)$ est composée d'espèces couramment commercialisées que sont: Akossika, Scottelia chevalieri (Flacourtiaceae); Bété, Mansonia altissima (Sterculiaceae); Fromager, Ceiba pentandra (Bombacaceae); Tali, Erythrophleum ivorense (Caesalpiniaceae); Amazakoué, Guibourtiaehie (Caesalpiniaceae); Fraké, Terminalia superba (Combretaceae); Kotibé, Nesogordonia papaverifera (Sterculiaceae), Aniégré blanc, Aningeria altissima (Sapotaceae); Koto, Pterygota macrocarpa (Sterculiaceae); Samba, Triplochiton scleroxylon (Sterculiaceae), Badi, Nauclea diderrichii (Rubiaceae), etc. La catégorie 2 (P2) est composée d'espèces sporadiquement commercialisées comme l'Asan, Celtis zenkeri (Ulmaceae); le Kékélé, Holoptelea grandis (Ulmaceae), le Lotofa, Sterculia rhinopetala (Sterculiaceae); le Tchebuessain, Xylia evansii(Mimosacea),leBa, Celtis mildbraedii (Ulmaceae); la Lohonfé , Celtis aldophi federici (Ulmaceae); le Pouo, Funtumia africana (Apocynaceae); l'Emien, Alstonia boonei (Apocynaceae), et le groupe P3 d'espèces à promouvoir.

Catalogue des Stations : Les 49 groupes statistiques ont été regroupés pour obtenir un catalogue des stations, plus utiles aux gestionnaires. Ainsi l'allure des peuplements, parce que facilement notable, et donc la typologie a été retenue pour définir huit grands types de stations. Des sous-types sont définis lorsque nécessaires pour tenir compte de l'impact local des conditions édaphiques sur la structure du peuplement. Un indice est alors ajouté à la lettre pour désigner la station $\left(C_{1}\right.$ par exemple). On obtient ainsi 22 types et sous- types de stations mis en évidence dans la Figure 4, plus informative lorsque superposée à la Figure 3 .

Cette dernière donne une description détaillée des stations en précisant entre autre, les groupes statistiques qui les composent, le niveau de contraintes édaphiques et le nombre de placeaux concernés. Les stations de type $H$, c'est-à-dire la forêt ruinée, constituent la majorité de la superficie de la forêt $(31,35 \%)$; les stations de type $E$ étant les moins fréquentes $(2,30 \%)$. Le catalogue des 22 types et soustypes de stations (Figure 5) donne pour chaque station, l'étendue en pourcent de la superficie de la forêt; ses groupes statistiques des placeaux de même que les conditions édaphiques qui la caractérisent. La structure du peuplement est donnée par l'effectif moyen des tiges des essences principales (P1), des essences secondaires et tertiaires (P23) et des autres essences (Autres) pour la régénération (REG), les peuplements d'avenir (AVE) et des tiges exploitables (EXP). 
Koula et al. J. Appl. Biosci. 2014. Polymorphie des associations au sein des forets : Déterminants, Catalogue des stations et cartographies complémentaires.

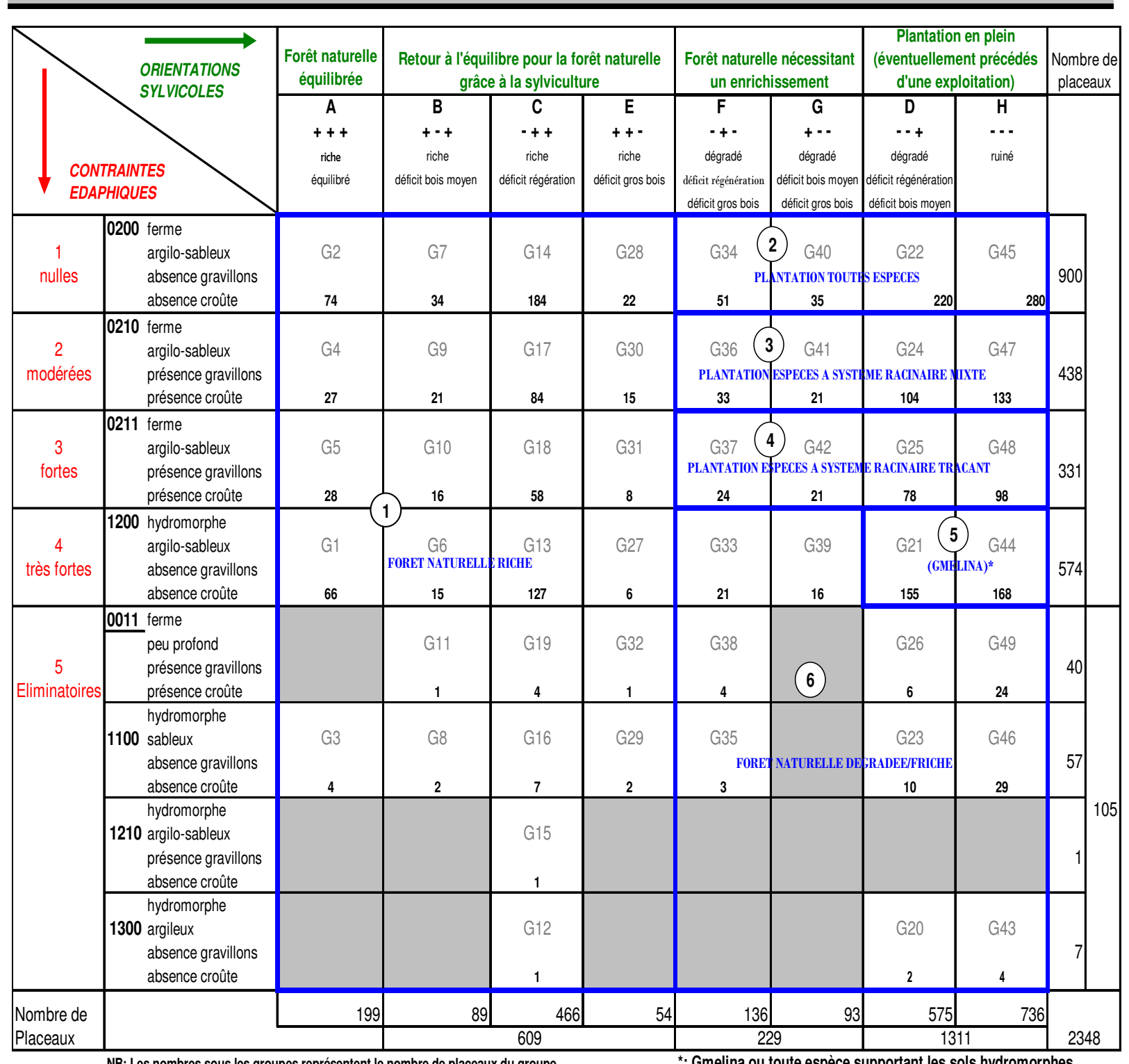

Figure 3 : Grille d'analyse et modalités de recommandations 
Koula et al. J. Appl. Biosci. 2014. Polymorphie des associations au sein des forets : Déterminants, Catalogue des stations et cartographies complémentaires.

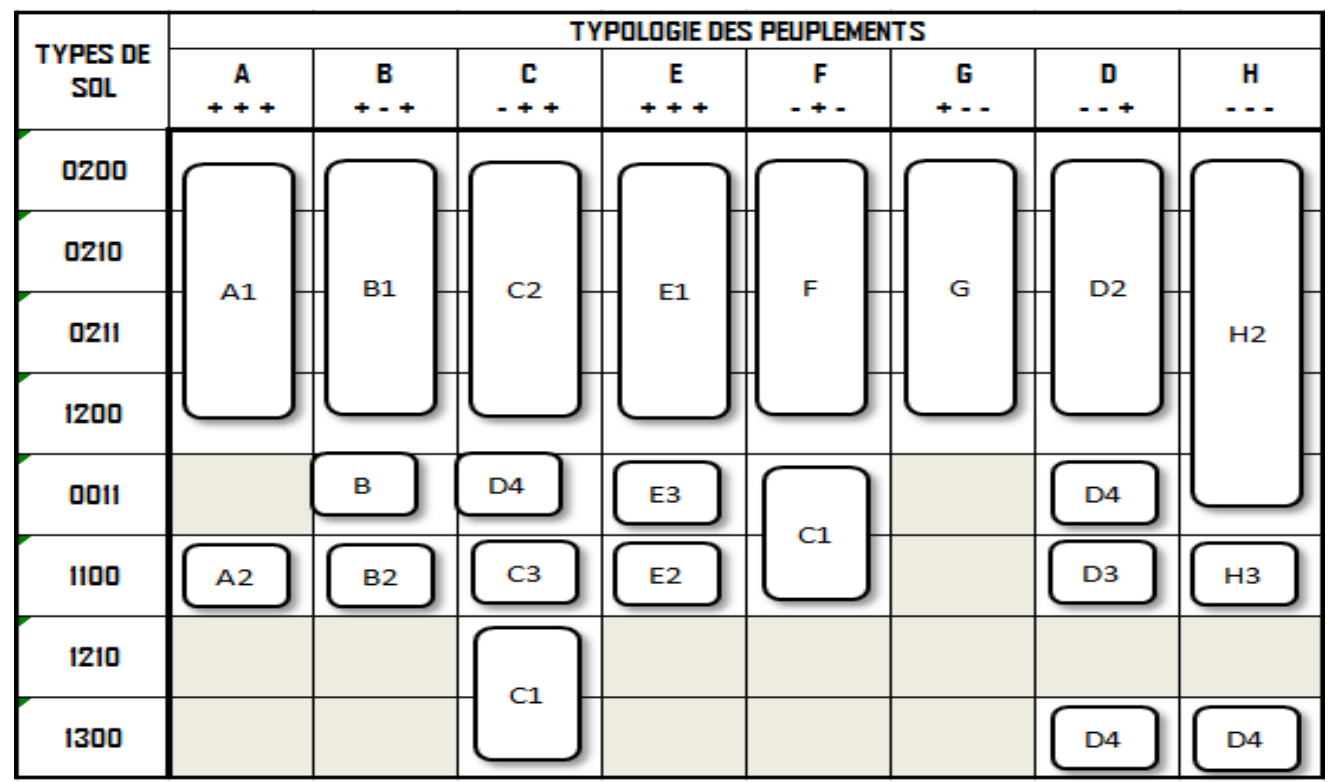

Figure 4 : Répartition des types de station

Carte des recommandations de mise en valeur : Les 6 strates cartographiques de la Figure 6 , traduction spatiale de la Figure 3, coïncident avec les 6types de recommandations. Elle révèle que plus du tiers de la superficie est encore couverte de forêt naturelle riche, cantonnée au nord du massif. Plus de la moitié (56\%) est très dégradée à ruinée. Seulement $5 \%$ des terres de la forêt n'a pas de vocation forestière ; $47 \%$ de la surface est apte à recevoir des travaux de reboisement à base d'espèces locales, auxquels s'ajoutent les $13 \%$ de terres hydromorphes où l'introduction en plein d'espèces locales ou exotiques adaptées est envisageable.

\begin{tabular}{|c|c|c|c|c|c|}
\hline STATION* & ESPECE & REG & AVE & EXP & GROUPES \\
\hline \multicolumn{6}{|c|}{ Type A : Peuplement riche et en équilibre } \\
\hline \multirow{3}{*}{$\begin{array}{c}A 1 \\
8,30 \%\end{array}$} & $\mathrm{P} 1$ & 272,90 & 51,86 & 16,78 & \multirow{3}{*}{$\begin{array}{l}\mathrm{G} 1, \mathrm{G} 2 \\
\mathrm{G} 4, \mathrm{G} 5\end{array}$} \\
\hline & P23 & 142,11 & 58,84 & 5,95 & \\
\hline & Autres & 5,81 & 50,76 & 1,22 & \\
\hline \multicolumn{6}{|c|}{ En majorité sols fermés argilo-sableux } \\
\hline \multirow{3}{*}{$\begin{array}{c}\text { A2 } \\
0,17 \%\end{array}$} & $\mathrm{P} 1$ & 175 & 43 & 15 & \multirow{3}{*}{ G3 } \\
\hline & P23 & 25 & 39,50 & 9 & \\
\hline & Autres & 0 & 48 & 1 & \\
\hline \multicolumn{6}{|c|}{ Sols hydromorphes argileux } \\
\hline \multicolumn{6}{|c|}{ Type B : Peuplement riche avec déficit en bois moyen } \\
\hline \multirow{3}{*}{$\begin{array}{c}\text { B1 } \\
3,66 \%\end{array}$} & $\mathrm{P} 1$ & 231,16 & 20,85 & 12,54 & \multirow{3}{*}{$\begin{array}{l}\mathrm{G} 6, \mathrm{G} 7 \\
\mathrm{G} 9, \mathrm{G} 10\end{array}$} \\
\hline & $\mathrm{P} 23$ & 327,22 & 70,20 & 13,67 & \\
\hline & Autres & 104,56 & 51,50 & 2,15 & \\
\hline \multicolumn{6}{|c|}{ En majorité sols fermes argilo-sableux } \\
\hline \multirow{3}{*}{$\begin{array}{c}\text { B2 } \\
0,09\end{array}$} & P1 & 300 & 22 & 8 & \multirow{3}{*}{ G8 } \\
\hline & P23 & 100 & 24 & 0 & \\
\hline & Autres & 0 & 20 & 0 & \\
\hline \multicolumn{6}{|c|}{ Sols sableux hydromorphes } \\
\hline \multirow{3}{*}{$\begin{array}{c}\text { B3 } \\
0,04\end{array}$} & P1 & 450 & 22 & 11 & \multirow[t]{3}{*}{ G11 } \\
\hline & P23 & 0 & 16 & 8 & \\
\hline & Autres & 0 & 24 & 3 & \\
\hline
\end{tabular}


Koula et al. J. Appl. Biosci. 2014. Polymorphie des associations au sein des forets : Déterminants, Catalogue des stations et cartographies complémentaires.

\begin{tabular}{|c|c|c|c|c|c|}
\hline \multicolumn{6}{|c|}{ Sols peu profonds, présence de gravillons et croûte latéritique } \\
\hline \multicolumn{6}{|c|}{ Type C : Peuplement riche avec déficit en régénération } \\
\hline \multirow{3}{*}{$\begin{array}{c}C 1 \\
0,09\end{array}$} & $\mathrm{P} 1$ & 0 & 81 & 12 & \multirow[t]{3}{*}{ G12, G15 } \\
\hline & P23 & 0 & 47 & 12 & \\
\hline & Autres & 0 & 53 & 1 & \\
\hline \multicolumn{6}{|c|}{ Sols fortement hydromorphes argilo-sableux } \\
\hline \multirow{3}{*}{$\begin{array}{c}\text { C2 } \\
19,29 \%\end{array}$} & P1 & 46,49 & 47,95 & 16,75 & \multirow{3}{*}{$\begin{array}{l}\text { G13, G14, } \\
\text { G17, G18 }\end{array}$} \\
\hline & P23 & 126,55 & 57,24 & 5,87 & \\
\hline & Autres & 40,83 & 51,51 & 1,18 & \\
\hline \multicolumn{6}{|c|}{ En majorité sols fermes argilo-sableux } \\
\hline \multirow{3}{*}{$\begin{array}{c}\text { C3 } \\
0,30 \% \\
\end{array}$} & P1 & 114,29 & 49,43 & 12,86 & \multirow{3}{*}{ G16 } \\
\hline & P23 & 71,43 & 38,86 & 8,00 & \\
\hline & Autres & 0,00 & 64,57 & 5,43 & \\
\hline \multicolumn{6}{|c|}{ Sols sableux hydromorphes } \\
\hline \multirow{3}{*}{$\begin{array}{c}\text { C4 } \\
0,17 \% \\
\end{array}$} & P1 & 0 & 46 & 11,50 & \multirow{3}{*}{ G19 } \\
\hline & $\mathrm{P} 23$ & 75 & 39 & 3 & \\
\hline & Autres & 50 & 44 & 1 & \\
\hline \multicolumn{6}{|c|}{ Sols fermes peu profonds, avec gravillons et croûte latéritiques } \\
\hline \multicolumn{6}{|c|}{\begin{tabular}{l}
\multicolumn{4}{|c|}{ Types D : Peuplement dégradé, déficit en régénération et bois moyens } \\
0
\end{tabular}} \\
\hline \multirow{3}{*}{$\begin{array}{c}\mathrm{D} 1 \\
0,09 \% \\
\end{array}$} & P1 & 0 & 20 & 11 & \multirow{3}{*}{ G20 } \\
\hline & $\mathrm{P} 23$ & 250 & 4 & 2 & \\
\hline & Autres & 0 & 29 & 1 & \\
\hline \multicolumn{6}{|c|}{ Sols argileux hydromorphes } \\
\hline \multirow{3}{*}{$\begin{array}{c}\text { D2 } \\
23,72 \% \\
\end{array}$} & P1 & 250 & 20 & 11 & \multirow{4}{*}{$\begin{array}{l}\mathrm{G} 21, \mathrm{G} 22, \\
\mathrm{G} 24, \mathrm{G} 25\end{array}$} \\
\hline & $\mathrm{P} 23$ & 250 & 4 & 2 & \\
\hline & Autres & 0 & 29 & 1 & \\
\hline & & rité sols fe & argilo-sab & & \\
\hline
\end{tabular}

\begin{tabular}{|c|c|c|c|c|c|}
\hline STATION* & ESPECE & REG & AVE & EXP & GROUPES \\
\hline \multirow{3}{*}{$\begin{array}{c}\text { D3 } \\
0,43 \% \\
\end{array}$} & P1 & 20 & 20,8 & 10,20 & \multirow{3}{*}{ G23 } \\
\hline & P23 & 50 & 12,6 & 3 & \\
\hline & Autres & 130 & 33,4 & 2,4 & \\
\hline \multicolumn{6}{|c|}{ Sols sableux hydromorphes } \\
\hline \multirow{3}{*}{ D4 $0,26 \%$} & $\mathrm{P} 1$ & 33,33 & 16,67 & 9,67 & \multirow{3}{*}{ G26 } \\
\hline & P23 & 0,00 & 14,00 & 2,67 & \\
\hline & Autres & 383,33 & 41,00 & 3,00 & \\
\hline \multicolumn{6}{|c|}{ Type E : Peuplement riche avec déficit en gros bois } \\
\hline \multirow{3}{*}{$\begin{array}{c}E 1 \\
2,17 \% \\
\end{array}$} & P1 & 294,20 & 40,59 & 3,96 & \multirow{3}{*}{$\begin{array}{l}\mathrm{G} 27, \mathrm{G} 28, \\
\mathrm{G} 30, \mathrm{G} 31\end{array}$} \\
\hline & P23 & 148,94 & 36,23 & 3,42 & \\
\hline & Autres & 22,54 & 47,07 & 0,71 & \\
\hline \multicolumn{6}{|c|}{ En majorité sols fermes argilo-sableux } \\
\hline \multirow{3}{*}{$\begin{array}{c}\text { E2 } \\
0,09 \% \\
\end{array}$} & P1 & 200 & 40,59 & 3,96 & \multirow{3}{*}{ G29 } \\
\hline & P23 & 0 & 6 & 1 & \\
\hline & Autres & 0 & 24 & 3 & \\
\hline \multicolumn{6}{|c|}{ Sols sableux hydromorphes } \\
\hline \multirow{3}{*}{$\begin{array}{c}E 3 \\
0,30 \% \\
\end{array}$} & P1 & 400 & 42 & 0 & \multirow{3}{*}{ G32 } \\
\hline & P23 & 0 & 36 & 2 & \\
\hline & Autres & 0 & 26 & 0 & \\
\hline & Sols & nds, pré & de grav & set cro & tique \\
\hline
\end{tabular}


Koula et al. J. Appl. Biosci. 2014. Polymorphie des associations au sein des forets : Déterminants, Catalogue des stations et cartographies complémentaires.

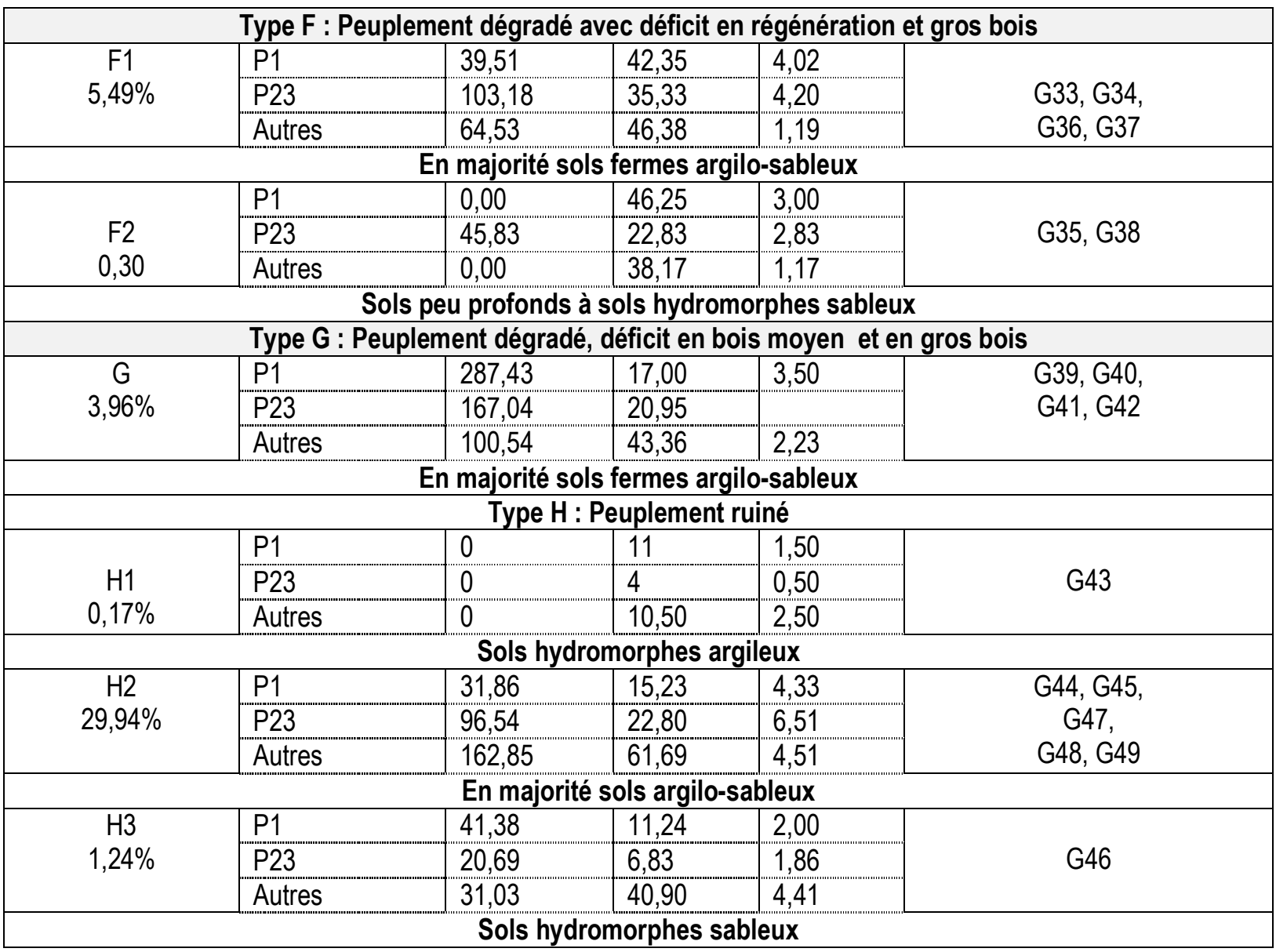

\section{* : Station suivie de la proportion de forêt occupée}

Figure 5 : Catalogue des stations

\section{CONCLUSION}

La gestion durable qui s'impose comme solution aux menaces sur un massif forestier nécessite sa connaissance approfondie. II a été ici proposé à cette fin une démarche qui dégage une typologie forestière très pragmatique qui débouche sur l'optimisation des actions sylvicoles depuis les éclaircies dans les peuplements naturelles jusqu'à la plantation pure assortie de certaines actions intermédiaires en fonction des cas de figure rencontrés. L'hétérogénéité de la forêt par rapport à son couvert végétal malgré son homogénéité par rapport aux types de sols même dans les zones sans culture implique des raisons autres que l'infiltration pour des besoins agricoles. 


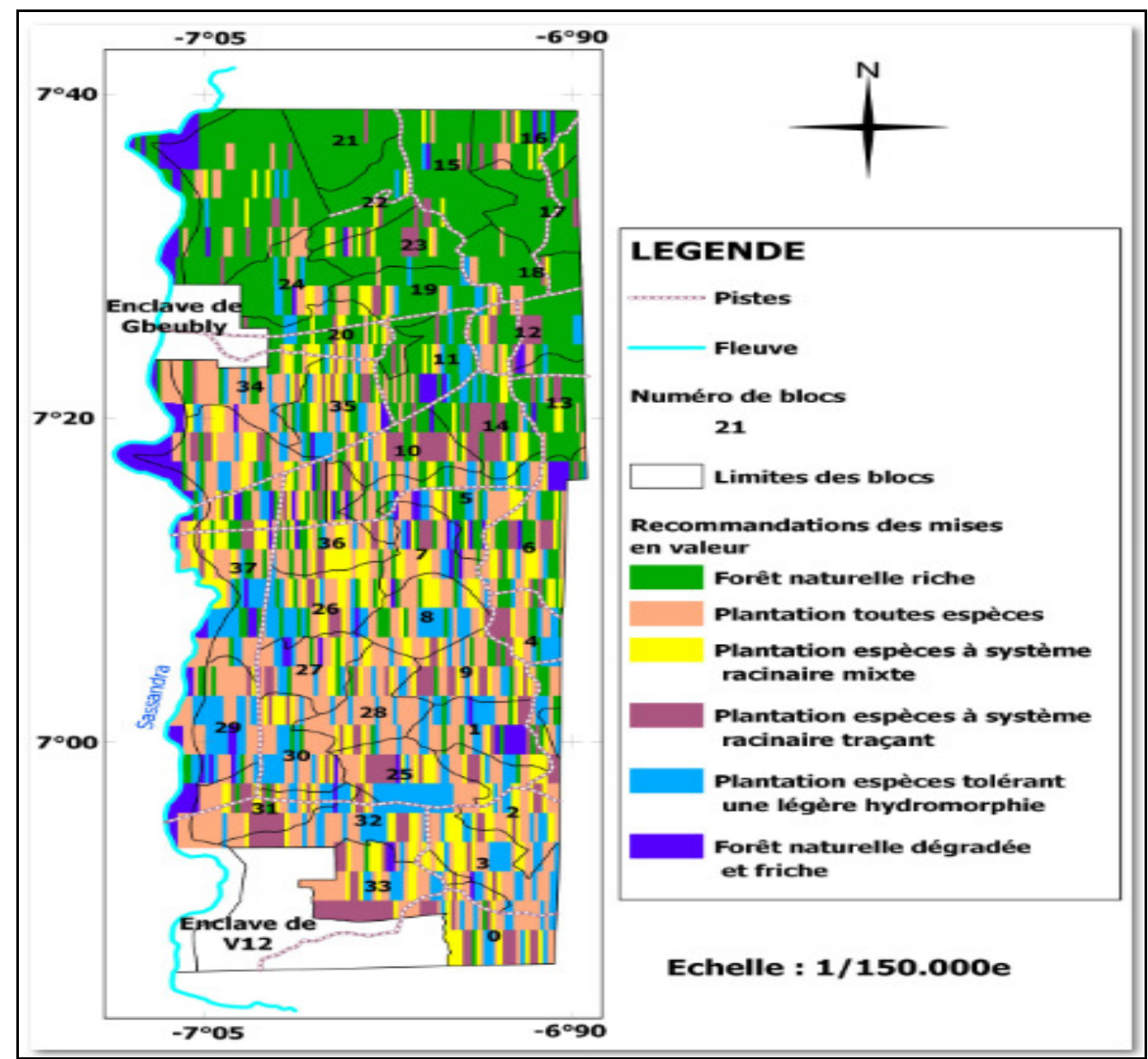

Figure 6 : Carte de recommandations de mise en valeur pour la forêt classée du Haut-Sassandra

Le reboiseur devra disposer de capacités matérielle et financière appréciables nécessaires à une bonne reconquête forestière. II pourra recourir à la reconstitution naturelle partout mais avec une présence dissuasive du reboiseur. Un système d'informations

\section{RÉFÉRENCES}

Gibily B. Le bois dans l'économie ivoirienne, Thèse de $3^{\text {ème }}$ cycle, Université de Bordeaux. (1970).

N'Guessan, E., N'Da, D. H., Bellane, M. F., Blasco, F. Pression anthropique sur une réserve forestière en Côte d'lvoire: Apport de la télédétection. Télédétection, (2006). Vol. 5, p.307-333.

Brou Yao, T., Servat, E. et Paturel, J.E. Contribution à l'analyse des interrelations entre activités humaine set variabilité climatique : cas du sud forestier ivoirien. Académie des sciences/ Elsevier, Paris, tome 327, série lla, (1999). p. 833-838. géographiques fréquemment mis à jour devrait permettre une meilleure maîtrise de la dynamique du peuplement et par conséquent un programme de gestion plus rationnelle.

N'Da, D.H., N'Guessan E.K., Wadja E.M., Affian K. Apport de la télédétection au suivi de la déforestation dans le Parc National de la Marahoué, Revue Télédétection, (2008), Vol. 8, n¹, p.17-34.

Kouamé, F. N. Influence de l'exploitation forestière sur la végétation et la flore de la Forêt Classée du Haut-Sassandra (Centre Ouest de la Côte d'lvoire). Thèse Doct. 3e Cycle, UFR Biosciences, Université de Cocody-Abidjan. (1998) $227 \mathrm{p}$.

Oswald J. Dynamique des formations agroforestières en Côte d'Ivoire (des années 1980 aux années 
Koula et al. J. Appl. Biosci. 2014. Polymorphie des associations au sein des forets : Déterminants, Catalogue des stations et cartographies complémentaires.

2000). Suivi par télédétection et
développement d'une approche
cartographique. Thèse de Doctorat de
géographie. Université des Sciences et
Techniques de Lille, 2005. 304. pp

Morisson, D. F. Multivariate Statistical Methods, $3^{\text {rd }}$ Edition, McGraw-Hill Inc. (1990)

Legendre, L. et Legendre, P. Ecologie numérique, Tome 2: La structure des données écologiques. Masson et les Presses de I'Université du Québec, Paris (1979). 330 p.

Ibanez, F. Effet des transformations des données dans l'analyse factorielle en écologie planctonique. Cah. Océanogr. (1971) 23, p.545-561.

Hartigan, J.A. Clustering algorithms. NewYork: John Willey \& sons. (1975).

Gower, J.C. A comparison of some methods of cluster analysis. Biometrics, 23, p. 623-632. (1967).

Wilkinson, L., Engelman, L., Corter, J., Coward, M. Cluster Analysis. In Systat: Statistics I. I-57-I91. (2004). 